\title{
THE METHOD OF TRANSMISSION MATRIX FOR INVESTIGATING PLANAR MECHANISMS
}

\author{
DO SANH \\ Hanoi University of Technology \\ DO DANG KHOA \\ University of Texas at Austin, USA
}

\begin{abstract}
In this paper the method of transmission matrix is applied for investigating planar mechanisms [5-7]. As is known, in many engineering problems, for example, in the problem of synthesis and analysis of motion, the following problem is more and more interested. There is an object (a particle or body) connected with a frame of reference, which moves with respect to another frame. It is necessary to define the configuration (the position, velocity, acceleration) of the object under consideration in the latter frame. There are two types of the frame of reference. A frame that is fixed in time named inertia frame or global frame of reference and the frame attached to each body of the system- the body frame of reference. The problem is stated now as follows: Determine the configuration in global frame of a particle fixed in any body of the system.

In this paper a method is constructed for solving such a problem by means of transmission matrices.

The obtained results are illustrated by examples.
\end{abstract}

\section{THE FORMULAS FOR DETERMINING THE CONFIGURATION OF THE OBJECT}

Let us consider two bodies of a free loop, which are numbered as $(i-1)$-body and $i$ body, joined together by the hinge $A_{i}$. Attach a frame of reference into each body (the body-frame). The ( $i-1)$ body - frame has the origin at $A_{i-1}$ and its axis $A_{i-1} x_{i-1}$ directing from $A_{i-1}$ to $A_{i}$, where $A_{i-1}$ is the hinge joining the $(i-1)$ body with the $(i-2)$ body.

The axis $A_{i-1} y_{i-1}$ is perpendicular to $A_{i-1} x_{i-1}$ in the direction of counter clockwise. The frame of reference glued to the i-body is formed in the similar way. The angle between $A_{i-1}$ two frames of reference denoted by $\varphi_{i}$, is the $\mathrm{M}$ rotating angle of the axis $A_{i-1} x_{i-1}$ to $A_{i} x_{i}$ in direction of counterclockwise (Fig. 1) [3, 7]. Let consider the point $M$ fixed on the $i$-body. The coordinates of the point $M$ in the $i$-body frame are denoted by the constants $a$ and $b$ and in the $(i-1)$ body frame - by $^{i-1} x_{M}$, ${ }^{i-1} y_{M}$, which are variables of time. The notation located at high left corner is number of the body, on which the particle $M$ on. Similarly, the coordinates of the point $M$ in the inertia reference is denoted by ${ }^{0} x_{M},{ }^{0} y_{M}$.

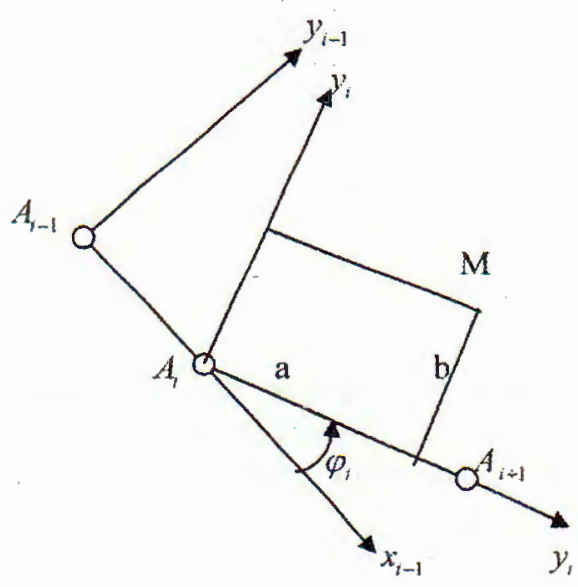

Fig. 1 
The components of the coordinate of the point $M$ in two frame in progressive order are related by the formula $[4,5,7]$

$$
\left\|\begin{array}{c}
{ }^{i-1} x_{M} \\
i-1 \\
{ }^{2} \\
1
\end{array}\right\|=\left\|\begin{array}{ccc}
\cos \varphi_{i} & -\sin \varphi_{i} & L_{i-1} \\
\sin \varphi_{i} & \cos \varphi_{i} & 0 \\
0 & 0 & 1
\end{array}\right\|\left\|\begin{array}{l}
a \\
a \\
1
\end{array}\right\|
$$

where: $L_{i-1} \equiv \overline{A_{i-1} A_{i}}$.

By introducing the symbols $\cos \varphi_{i} \equiv c_{i}, \sin \varphi_{i} \equiv s_{i}$ and the matrices

$$
\begin{aligned}
& T_{i} \equiv\left\|\begin{array}{ccc}
\cos \varphi_{i} & -\sin \varphi_{i} & L_{i-1} \\
\sin \varphi_{i} & \cos \varphi_{i} & 0 \\
0 & 0 & 1
\end{array}\right\| \equiv\left\|\begin{array}{ccc}
c_{i} & -s_{i} & L_{i-1} \\
s_{i} & c_{i} & 0 \\
0 & 0 & 1
\end{array}\right\| . \\
& { }^{i-1} r^{T}=\left\|{ }^{i-1} x_{M} \quad{ }^{i-1} y_{M} \quad 1\right\|, \quad{ }^{i} r^{T}=\|a \quad b \quad 1\|
\end{aligned}
$$

the formula (1.1) is written in the form

$$
{ }^{i-1} r=T_{i}^{i} r .
$$

Hereafter the matrix is noted by the bold letter and the vector is identify as $a(3 \times 1)$ matrix. Let us consider a system of $k$ bodies in plane (where $k=3$ ), which are joined between each other by the hinges $A_{1}, A_{2}, \ldots, A_{k}$ respectively, where $A_{1}$ is fixed at time. Let put the inertia frame of reference at $A_{1}$. For simplicity instead of the $i$-body frame let us put the vector $x_{i}$ with the origin at $A_{i}$ and directing along the axis $A_{i} x_{i}$. The angle between two vectors $x_{i-1}$ and $x_{i}$ (Fig. 2). It is easy to derive the relationship between the coordinates of the point fixed to the $k$-body frame (with the coordinates $a, b$ ) and its coordinates in the global reference, that is

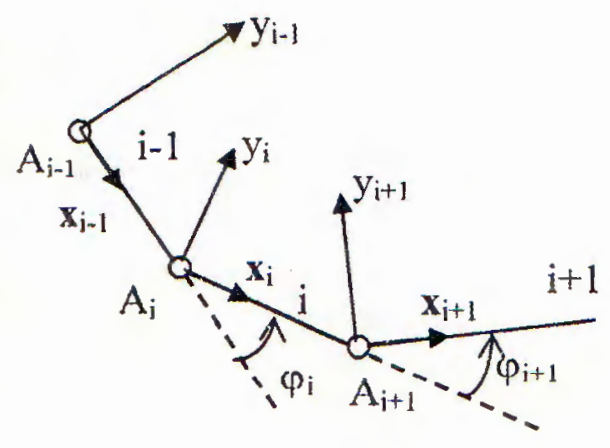

Fig. 2

$$
\left\|\begin{array}{c}
{ }^{0} x \\
0 \\
y \\
1
\end{array}\right\|=\mathbf{T}_{\mathbf{1}} \mathbf{T}_{\mathbf{2}} \ldots \mathbf{T}_{\mathbf{k}}\left\|\begin{array}{c}
a \\
b \\
1
\end{array}\right\|
$$

or

$$
{ }^{0} \mathbf{r}_{M}=\mathbf{T}_{\mathbf{1}} \mathbf{T}_{2} \ldots \mathbf{T}_{\mathbf{k}} \mathbf{r}_{M}
$$

where the matrices $\mathbf{T}_{i}\left(\overline{1, k)}\right.$ take the same form as $(1.2)$ in consideration of $L_{0}=0$.

By the formulas(1.5) and (1.6) we calculate the components of velocity of the point $M$ in the global reference, that is

$$
\boldsymbol{v}=\dot{\mathbf{r}}=\left\|\begin{array}{c}
0 \dot{x}_{M} \\
{ }^{0} \dot{y}_{M} \\
0
\end{array}\right\|=\sum_{i=1}^{k} \dot{\varphi}(*) v_{i}
$$


where $\dot{\varphi}_{i}(*)$ is $a(3 \times 3)$ diagonal matrix of the form

$$
\dot{\varphi}_{i}(*)=\left\|\begin{array}{ccc}
\dot{\varphi}_{i} & 0 & 0 \\
0 & \dot{\varphi}_{i} & 0 \\
0 & 0 & \dot{\varphi}_{i}
\end{array}\right\|
$$

and the matrixces $v_{i}(i=\overline{1, k)}$ are calculated by following the formula

$$
v_{i}=\mathbf{T}_{\mathbf{1}} \mathbf{T}_{\mathbf{2}} \ldots \mathbf{T}_{\mathbf{i}}^{\prime} \ldots \mathbf{T}_{k},
$$

in which the matrices $\mathbf{T}_{i}$ take the form (1.2), but the matrices $\mathbf{T}_{i}^{\prime}$ are drawn as follows

$$
\left\|\mathbf{T}_{i}^{\prime}\right\|=\left\|\begin{array}{ccc}
-s_{i} & -c_{i} & 0 \\
c_{i} & -s_{i} & 0 \\
0 & 0 & 0
\end{array}\right\|
$$

It is easy to show that the absolute angular velocity of the $k^{\text {th }}$ link is calculated by the formula

$$
\Omega_{k}=\sum_{i=1}^{k} \dot{\varphi}_{i}
$$

The components of acceleration of the point $M$ in the global frame of reference are determined by following formula

$$
\mathbf{a}=\left\|\begin{array}{c}
0 \\
{ }^{\dot{x}_{M}} \\
0 \dot{y}_{M} \\
0
\end{array}\right\|=\sum_{i=1}^{k} \ddot{\varphi}_{i}(*) \boldsymbol{v}_{i}+\sum_{i=1}^{k} \dot{\varphi}_{i}(*) \boldsymbol{v}_{i}^{\prime}+2 \sum_{i=1}^{k-1} \sum_{j>i} \dot{\varphi}_{i}(*) \dot{\varphi}_{j}(*) \boldsymbol{v}_{i, j},
$$

where $v_{i}$ is calculated by $(1.9), \ddot{\varphi}(*)$ is a $(3 \times 3)$ diagonal matrix of the form

$$
\ddot{\varphi}_{i}(*)=\left\|\begin{array}{ccc}
\ddot{\varphi}_{i} & 0 & 0 \\
0 & \ddot{\varphi}_{i} & 0 \\
0 & 0 & \ddot{\varphi}_{i}
\end{array}\right\|
$$

but $\boldsymbol{v}_{i}^{\prime}, \boldsymbol{v}_{i, j}$ are determined by following formulas

$$
\begin{gathered}
v_{i}^{\prime}=\mathbf{T}_{\mathbf{1}} \mathbf{T}_{2} \ldots \mathbf{T}_{i \ldots}^{\prime \prime} \mathbf{T}_{k}, \\
v_{\mathbf{i}, \mathbf{j}}=\mathbf{T}_{\mathbf{1}} \mathbf{T}_{\mathbf{2}} \ldots \mathbf{T}_{\mathbf{i}}^{\prime} \ldots \mathbf{T}_{\mathbf{j}}^{\prime} \ldots \mathbf{T}_{k},
\end{gathered}
$$

where

$$
T_{i}^{\prime \prime}=\left\|\begin{array}{ccc}
-c_{i} & s_{i} & 0 \\
-s_{i} & -c_{i} & 0 \\
0 & 0 & 0
\end{array}\right\|
$$

The absolute angular acceleration of the $k^{\text {th }}$ link is of the form

$$
\overline{\varepsilon_{k}}=\sum_{i=1}^{k} \ddot{\varphi}_{i} \text {. }
$$

In order to use the above obtained formulas it is necessary to bring to the following notices

a) In the case of an open loop, i.e. when the last link of the loop under consideration is one end free, it is possible to apply directly these formulas 
b) In the case of closed loop, i.e. there is a point of the last link fixed at time with respect to the global frame of reference, first it is necessay to find the configuration of this point.

By such a way we get two equations for each kind of the configuration (the position, velocity, acceleration). Solving the obtained equations we determine the configuration of the system under consideration.

c) When the system is a semi-closed loop, i.e. when a point of the last link moves along any curve fixed in the global frame of reference, it is necessary to write the configuration equations for this point, by which we will find the configuration of the system.

\section{APPLICATION}

Example 1. Let us consider a free loop of three links (Fig. 3). We put three body frames of reference as in the Fig. $3[5,6]$, which are expressed by three vectors $x_{1}, x_{2}, x_{3}$. The global frame of reference is noted by the vector $x_{0}$. By such a way there are three transmission matrices, that are

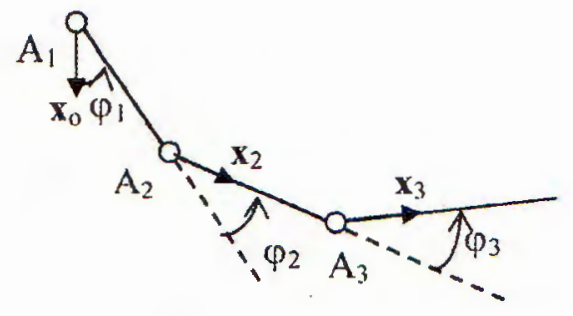

Fig. 3

$$
T_{1}=\left\|\begin{array}{ccc}
c_{1} & -s_{1} & 0 \\
s_{1} & c_{1} & 0 \\
0 & 0 & 1
\end{array}\right\| ; T_{2}=\left[\left\|\begin{array}{ccc}
c_{2} & -s_{2} & L_{1} \\
s_{2} & c_{2} & 0 \\
0 & 0 & 1
\end{array}\right\|\right] \quad T_{2}=\left\|\begin{array}{ccc}
c_{3} & -s_{3} & L_{2} \\
s_{3} & c_{3} & 0 \\
0 & 0 & 1
\end{array}\right\|
$$

In accordance with the rules $(1.10),(1.16)$ we have

$$
\begin{gathered}
T_{1}=\left\|\begin{array}{ccc}
-s_{1} & -c_{1} & 0 \\
c_{1} & -s_{1} & 0 \\
0 & 0 & 0
\end{array}\right\| ; \quad T_{2}^{\prime}=\left\|\begin{array}{ccc}
-s_{2} & -c_{2} & 0 \\
c_{2} & -s_{2} & 0 \\
0 & 0 & 0
\end{array}\right\| ; \quad T_{3}^{\prime}=\left\|\begin{array}{ccc}
-s_{3} & -c_{3} & 0 \\
c_{3} & -s_{3} & 0 \\
0 & 0 & 0
\end{array}\right\| . \\
T_{1}^{\prime \prime}=\left\|\begin{array}{ccc}
-c_{1} & s_{1} & 0 \\
-s_{1} & -c_{1} & 0 \\
0 & 0 & 0
\end{array}\right\| ; T_{2}^{\prime \prime}=\left\|\begin{array}{ccc}
-c_{2} & s_{2} & 0 \\
-s_{2} & -c_{2} & 0 \\
0 & 0 & 0
\end{array}\right\| ; \quad T_{3}^{\prime \prime}=\left\|\begin{array}{ccc}
-c_{3} & s_{3} & 0 \\
-s_{3} & -c_{3} & 0 \\
0 & 0 & 0
\end{array}\right\| .
\end{gathered}
$$

Let us consider the point $M$ fixed in the $3^{\text {th }}$ - link and having the body-coordinates to be $a$ and $b$. The components of coordinate of the point $M$ in the global reference are computed by following the formula (1.5), that are

$$
\left\|\begin{array}{c}
{ }^{0} x_{M} \\
{ }^{0} y_{M} \\
1
\end{array}\right\|=\mathbf{T}_{\mathbf{1}} \mathbf{T}_{\mathbf{2}} \mathbf{T}_{\mathbf{3}}\left\|\begin{array}{l}
a \\
b \\
1
\end{array}\right\|=\left\|\begin{array}{c}
a c_{123}-b s_{123}+L_{2} c_{12}+L_{1} c_{1} \\
a s_{123}+b c_{123}+L_{2} s_{12}+L_{1} s_{1} \\
1
\end{array}\right\|
$$

The coordinates of the point $M$ in the global reference are of the form

$$
{ }^{0} x_{M}=a c_{123}-b s_{123}+L_{2} c_{12}+L_{1} c_{1} ;{ }^{0} y_{M}=a s_{123}+b c_{123}+L_{2} s_{12}+L_{1} s_{1},
$$

where the following symbols are used

$c_{123} \equiv \cos \left(\varphi_{1}+\varphi_{2}+\varphi_{3}\right) ; s_{123} \equiv \sin \left(\varphi_{1}+\varphi_{2}+\varphi_{3}\right) ; c_{12} \equiv \cos \left(\varphi_{1}+\varphi_{2}\right) ; s_{12} \equiv \sin \left(\varphi_{1}+\varphi_{2}\right)$. 
In order to calculate the components of the velocity of the point $M$ in the global reference, let us write the formula (1.7). For this purpose we calculate the matrices $v_{1}, v_{2}, v_{3}$ by means of formula (1.9), that are

$$
\begin{aligned}
& \boldsymbol{v}_{1}=\boldsymbol{T}_{1}^{\prime} \boldsymbol{T}_{2} \boldsymbol{T}_{3} r=\left\|\begin{array}{c}
-a s_{123}-b c_{123}-\left(L_{2} s_{12}+L_{1} s_{1}\right) \\
a c_{123}-b s_{123}+L_{2} c_{12}+L_{1} c_{1} \\
0 \\
\boldsymbol{v}_{2}=T_{1} T_{2}^{\prime} \boldsymbol{T}_{3} r=\| \\
\begin{array}{c}
-\left(a s_{123}+b c_{123}+L_{2} s_{12}\right) \\
a c_{123}-b s_{123}+L_{2} c_{12} \\
0
\end{array} \| ; \\
\boldsymbol{v}_{3}=T_{1} \boldsymbol{T}_{2} \boldsymbol{T}_{3}^{\prime} r=\| \\
-\left(a s_{123}+b c_{123}\right) \\
a c_{123}-b s_{123}
\end{array}\right\| .
\end{aligned}
$$

The formula (1.7) takes the form now

$v=\left\|\begin{array}{c}0 \\ 0 \\ \dot{x}_{M} \\ \dot{y}_{M} \\ 1\end{array}\right\|=\left\|\begin{array}{c}-\left(a s_{123}+b c_{123}+L_{2} s_{12}+L_{1} s_{1}\right) \dot{\varphi}_{1}-\left(a s_{123}+b c_{123}+L_{2} s_{12}\right) \dot{\varphi}_{2}-\left(a s_{123}+b c_{123}\right) \dot{\varphi}_{3} \\ \left(a c_{123}-b s_{123}+L_{2} c_{12}+L_{1} c_{1}\right) \dot{\varphi}_{1}+\left(a c_{123}-b s_{123}+L_{2} c_{12}\right) \dot{\varphi}_{2}+\left(a c_{123}-b s_{123}\right) \dot{\varphi}_{3} \\ 0\end{array}\right\|$.

The components of acceleration of the point $M$ in the global reference are computed by the formula (1.12), where the matrices $v_{1}, v_{2}, v_{3}$ are determined as above, but the matrices $\boldsymbol{v}_{i}^{\prime}(i=1,2,3), \boldsymbol{v}_{i, j}(i, j=1,2,3)$ by means (1.14), (1.15) will be

$$
\begin{aligned}
& v_{1}^{\prime}=T_{1}^{\prime \prime} T_{2} T_{3} r=\left\|\begin{array}{c}
-\left(a c_{123}-b s_{123}+L_{2} c_{12}+L_{1} c_{1}\right) \\
-\left(a s_{123}+b c_{123}+L_{2} s_{12}+L_{1} s_{1}\right) \\
0
\end{array}\right\| \\
& \boldsymbol{v}_{2}^{\prime}=\boldsymbol{T}_{1} \boldsymbol{T}_{2}^{\prime} \boldsymbol{T}_{3} \boldsymbol{r}=\left\|\begin{array}{c}
-\left(a c_{123}-b s_{123}+L_{2} c_{12}\right) \\
-\left(a s_{123}+b c_{123}+L_{2} s_{12}\right) \\
0
\end{array}\right\| \\
& \boldsymbol{v}_{3}^{\prime}=T_{1} T_{2} T_{2}^{\prime \prime} \boldsymbol{r}=\left\|\begin{array}{c}
-a c_{123}+b s_{123} \\
-\left(a s_{123}+b c_{123}\right) \\
0
\end{array}\right\| \text {; } \\
& \boldsymbol{v}_{1,2}=\boldsymbol{T}_{1}^{\prime} \boldsymbol{T}_{2}^{\prime} \boldsymbol{T}_{3} \boldsymbol{r}=\left\|\begin{array}{c}
-\left(a c_{123}+b s_{123}+L_{2} c_{12}\right) \\
-\left(a s_{123}+b c_{123}+L_{2} s_{12}\right) \\
0
\end{array}\right\| \text {; } \\
& \boldsymbol{v}_{1,3}=\boldsymbol{T}_{1}^{\prime} \boldsymbol{T}_{2} \boldsymbol{T}_{3}^{\prime} \boldsymbol{r}=\left\|\begin{array}{l}
-\left(a c_{123}+b s_{123}\right) \\
-a s_{123}-b c_{123} \\
0
\end{array}\right\| ; \\
& \boldsymbol{v}_{2,3}=\boldsymbol{T}_{1} \boldsymbol{T}_{2}^{\prime} \boldsymbol{T}_{3}^{\prime} \boldsymbol{r}=\left\|\begin{array}{c}
-\left(a c_{123}+b s_{123}\right) \\
-\left(a s_{123}+b c_{123}\right) \\
0
\end{array}\right\| \text {. }
\end{aligned}
$$


By applying the (1.12) it is easy to find the components of acceleration of the point $M$ in the global reference, that are

$$
\begin{aligned}
{ }^{0} \ddot{x}_{M}= & -\left(a s_{123}+b c_{123}+L_{2} s_{12}+L_{1} s_{1}\right) \ddot{\varphi}_{1}+\left(a s_{123}+b c_{123}+L_{2} s_{12}\right) \ddot{\varphi}_{2} \\
& +\left(a s_{123}+b c_{123}+L_{2} s_{12}\right) \ddot{\varphi}_{3}-2\left(a c_{123}-b s_{123}+L_{2} c_{12}+L_{1} c_{1}\right) \dot{\varphi}_{1}^{2} \\
& -\left(a c_{123}-b s_{123}+L_{2} c_{12}\right) \dot{\varphi}_{2}^{2}-\left(a c_{123}+b s_{123}\right) \dot{\varphi}_{3}^{2}-2\left(a c_{123}+b s_{123}-L_{2} c_{12}\right) \dot{\varphi}_{1} \dot{\varphi}_{2} \\
& -2\left(a c_{123}-b s_{123}\right) \dot{\varphi}_{1} \dot{\varphi}_{3}-2\left(a c_{123}+b s_{123}\right) \dot{\varphi}_{2} \dot{\varphi}_{3} . \\
{ }^{0} \ddot{y}_{M}= & \left(a c_{123}-b s_{123}+L_{2} c_{12}+L_{1} c_{1}\right) \ddot{\varphi}_{1}+\left(a c_{123}-b s_{123}+L_{2} c_{12}\right) \ddot{\varphi}_{2}+\left(a s_{123}+b c_{123}\right) \ddot{\varphi}_{3} \\
& -\left(a s_{123}+b c_{123}+L_{2} s_{12}+L_{1} s_{1}\right) \dot{\varphi}_{1}^{2}-\left(a s_{123}+b c_{123}^{\prime} \dot{\varphi}_{2}^{2}-\left(a s_{123}+b c_{123}\right) \dot{\varphi}_{3}^{2}\right. \\
& -2\left(a s_{123}+b c_{123}+L_{2} s_{12}\right) \dot{\varphi}_{1} \dot{\varphi}_{2}-2\left(a s_{123}+b c_{123}\right) \dot{\varphi}_{1} \dot{\varphi}_{3}-2\left(a s_{123}+b c_{123}\right) \dot{\varphi}_{2} \dot{\varphi}_{3} .
\end{aligned}
$$

Of course, in the case of using numerical methods, it is unecessary to write the expressions in an universal form, for example, in the case of a loop of many links.

Example 2. Let us consider a slider-crank mechanism. The crank $O A$ rotates about $O$ with angular velocity and acceration $\omega_{1}$ and $\varepsilon_{1}$, respectively. The length of the crank $O A$ is $r=0.4 \mathrm{~m}$, but the one of the connecting $\operatorname{rod} A B$ is $L=2 \mathrm{~m}$. Determine the angular velocity and acceleration of the connecting rod at $\varphi=\frac{\pi}{2}$ (Fig. 4).

The mechanism under consideration is a semiclosed loop, because the point of the link $\mathrm{AB}$ (last link) moves along the horizontal line, fixed in the

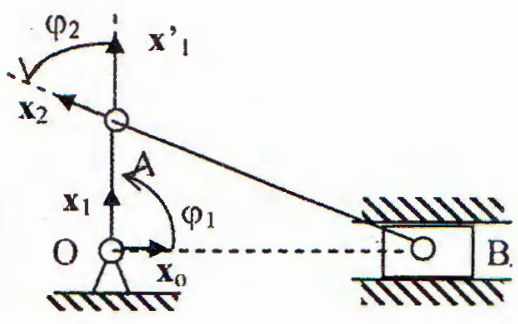

Fig. 4 inertia frame of reference. The system consists of two links joined each other by the hinge $A$. The global reference is represented by vector $x_{0}$ in horizontal direction to right side. The body-frames are represented by the vectors $x_{1}, x_{2}, x_{3}$ as shown in Fig, 4 . The point $B$ located at the second link. Its body-coordinates are $(-2,0)$, but the global coor-dinates are $\left(x_{B}, 0\right)$. In order to write the configuration equationsfor the considered system it is necessary to determined the transmission matrices $T_{1}, T_{2}, T_{1}^{\prime}, T_{2}^{\prime}, T_{1}^{\prime \prime}, T_{2}^{\prime \prime}$, which are of the form

$$
\begin{aligned}
& T_{1}=\left\|\begin{array}{ccc}
c_{1} & -s_{1} & 0 \\
s_{1} & c_{1} & 0 \\
0 & 0 & 1
\end{array}\right\| \\
& T_{2}=\left\|\begin{array}{ccc}
c_{2} & -s_{2} & 0.4 \\
s_{2} & c_{2} & 0 \\
0 & 0 & 1
\end{array}\right\| \\
& T_{1}^{\prime}=\left\|\begin{array}{ccc}
-s_{1} & -c_{1} & 0 \\
c_{1} & -s_{1} & 0 \\
0 & 0 & 0
\end{array}\right\| \\
& T_{2}^{\prime}=\left\|\begin{array}{ccc}
-s_{2} & -c_{2} & 0 \\
c_{2}-s_{2} & 0 & \\
0 & 0 & 0
\end{array}\right\| \\
& T_{1}^{\prime \prime}=\left\|\begin{array}{ccc}
-c_{1} & s_{1} & 0 \\
-s_{1} & -c_{1} & 0 \\
0 & 0 & 0
\end{array}\right\| \\
& T_{2}^{\prime \prime}=\left\|\begin{array}{ccc}
-c_{2} & s_{2} & 0 \\
-s_{2} & -c_{2} & 0 \\
0 & 0 & 0
\end{array}\right\|
\end{aligned}
$$

At the given position of the mechanism we have: $\varphi_{1}=\frac{\pi}{2}$. Therefore: $c_{1}=0 ; s_{1}=1$. In order to determine the position of the system at given time, i.e. the value of the angle $\varphi_{2}^{*}$, let us write the equation (1.5) for the point $B$ in consideration of the above 
calculated values, that are:

$$
\left\|\begin{array}{c}
0 \\
x_{B}^{*} \\
0 \\
1
\end{array}\right\|=T_{1}^{*} T_{2}^{*} \boldsymbol{r}=\left\|\begin{array}{c}
2 s_{2}^{*} \\
-2 c_{2}^{*}+0.4 \\
1
\end{array}\right\|
$$

Hereafter the symbol "*" located on high right corner of the letter denotes the value of the quantity at the given instant of time.

The obtained matrix equation is equivalent to following two equations:

$$
{ }^{0} x_{B}^{*}=-2 s_{2}^{*} ; \quad-2 c_{2}^{*}+0.4=0 .
$$

The angle $\varphi_{2}^{*}$ is defined by $c_{2}^{*}=0.2 ; s_{2}^{*}=\sqrt{0.96}$, but the coordinates of the point $B$ at the given instant of time is equal to $(2 \sqrt{0.96} ; 0)$.

For solving the problem of velocity, we apply the formula (1.7). First let us to calculate the matrices $\boldsymbol{v}_{1}, \boldsymbol{v}_{2}$ at the given time, that are

$$
v_{1}^{*}=T_{1}^{*} T_{2}^{*} r=\left\|\begin{array}{c}
0 \\
2 \sqrt{0.96} \\
0
\end{array}\right\| ; \quad v_{2}^{*}=T_{1}^{*} T_{2}^{\prime *} r=\left\|\begin{array}{c}
0.4 \\
2 \sqrt{0.96} \\
0
\end{array}\right\|
$$

By means of (1.7) we calculate

$$
{ }^{0} \dot{x}_{B}^{*}=0.4 \dot{\varphi}_{1}^{*}=0.4 \omega_{1} ; \quad{ }^{0} \dot{y}_{B}^{*}=2 \sqrt{0.96} \dot{\varphi}_{1}^{*}+2 \sqrt{0.96} \dot{\varphi}_{2}^{*} .
$$

Because the velocity of the point $B$ is in horizontal direction, i.e. ${ }^{0} \dot{y}_{B}^{*}=0$, we have then

$$
0=\dot{\varphi}_{1}^{*}+\dot{\varphi}_{2}^{*} \rightarrow \dot{\varphi}_{2}^{*}=-\dot{\varphi}_{1}^{*} .
$$

Absolute angular velocity $\Omega_{2}$ of the connecting rod $A B$ is determined by means of (1.11):

$$
\Omega_{2}^{*}=\dot{\varphi}_{1}^{*}+\dot{\varphi}_{2}^{*}=0 .
$$

This means that at the given instant of time the rod $A B$ is in instantaneous translation.

The velocity of the point $B$ is equal to

$$
v_{B}^{*} \equiv{ }^{0} \dot{x}_{B}^{*}=0.4 \dot{\varphi}_{1}^{*}=r \omega_{1}=v_{A}^{*} .
$$

It is clear, because the rod $A B$ is in instantaneous translation.

In order to determine acceleration it is necessary to write the equation (1.12). For this aim, let us calculate the matrices $\boldsymbol{v}_{1}^{\prime}, \boldsymbol{v}_{2}^{\prime}, \boldsymbol{v}_{1,2}$ corresponding to the given of time. That are $\boldsymbol{v}_{1}^{\prime *}=\boldsymbol{T}_{1}^{\prime \prime}{ }_{1}^{*} \boldsymbol{T}_{2}^{*} \boldsymbol{r}=\left\|\begin{array}{c}-2 \sqrt{0.96} \\ 0 \\ 0\end{array}\right\| ; \boldsymbol{v}_{2}^{*}=\boldsymbol{T}_{1}^{*} \mathbf{T}_{2}^{\prime \prime}{ }_{2} \mathbf{r}=\left[\begin{array}{c}-2 \sqrt{0.96} \\ 0.4 \\ 0\end{array}\right] ; \boldsymbol{v}_{1,2}^{*}=\mathbf{T}_{1}^{*} \mathbf{T}^{\prime \prime *}{ }_{2} \mathbf{r}=\left\|\begin{array}{c}-2 \sqrt{0.96} \\ 0.4 \\ 0\end{array}\right\|$.

By writting and developping the equation (1.12) the components of acceleration of the point $B$ in the global reference will be

$$
\begin{aligned}
& { }^{0} \ddot{x}_{B}^{*}=0.4 \ddot{\varphi}_{2}^{*}-2 \sqrt{0.96} \dot{\varphi}_{1}^{2 *}-2 \sqrt{0.96} \dot{\varphi}_{2}^{2 *}-4 \sqrt{0.96} \dot{\varphi}_{1}^{*} \dot{\varphi}_{2}^{*} \\
& { }^{0} \ddot{y}_{B}^{*}=2 \sqrt{0.96} \ddot{\varphi}_{1}^{*}+2 \sqrt{0.96} \ddot{\varphi}_{2}^{*}+0.4 \dot{\varphi}_{2}^{2 *}+0.8 \dot{\varphi}_{1}^{*} \dot{\varphi}_{2}^{*}=0
\end{aligned}
$$

Solving the last equation in consideration of $\dot{\varphi}_{2}^{*}=-\dot{\varphi}_{1}^{*}$, we obtain

$$
\ddot{\varphi}_{2}^{*}=-\ddot{\varphi}_{1}^{*}+\frac{0.2}{\sqrt{0.96}} \dot{\varphi}_{1}^{2 *}
$$


The absolute angular acceleration of the $\operatorname{rod} A B$ are of the form

$$
\varepsilon_{2}^{*}=\ddot{\varphi}_{1}^{*}+\ddot{\varphi}_{2}^{*}=\frac{0.2}{\sqrt{0.96}} \dot{\varphi}_{1}^{2 *}=\frac{0.2}{\sqrt{0.96}} \omega_{1}^{2} .
$$

It is easy to find the acceleration of the point $B$ at the given instant of time in the global reference

$$
a_{B}^{*}={ }^{0} \ddot{x}_{B}^{*}=-0.4 \ddot{\varphi}_{1}^{*}+\frac{0.08}{\sqrt{0.96}} \dot{\varphi}_{1}^{2 *}=-0.4 \varepsilon_{1}+\frac{0.08}{\sqrt{0.96}} \omega_{1}^{2} .
$$

Example 3. Let consider a four bar mechanism. The rod $A B$ and $D C$ are same length $R=0.4 \mathrm{~m}$. The length of the connecting rod $B C$ is $L=0.2 \mathrm{~m}$ Determinee angular velocity and acceleration of the connecting rod $B C$ when the mechanism takes the position shown in the Fig. 5 . At the given instant of time the crank $A B$ rotates about $A$ with the angular velocity and acceleration $\omega_{1}, \varepsilon_{1}$, respectively. The system under consideration consits of three moving bars. This is a closed loop because the point $D$ of the last link is fixed in the inertia reference. Let us choose the global reference represented by the vector $x_{0}$, having the origin at $A$ and directing to the right side. The body frames of reference are chosen as in Fig. 5. At the interest position of mechanism we have

$$
\varphi_{2}=\frac{\pi}{2}, \quad \varphi_{1}+\varphi_{3}=\pi \rightarrow c_{2}=0, \quad s_{2}=1, \quad c_{3}=-c_{1}, \quad s_{3}=s_{1} .
$$

Let us write the configuration equation for the point $D$, which has the global and body coordinates are $(0.2,0)$ and $(0.4,0)$ respectively. "For this aim let us calculate the following matrices

$$
\begin{gathered}
T_{1}^{*}=\left\|\begin{array}{ccc}
c_{1}^{*} & -s_{1}^{*} & 0 \\
s_{1}^{*} & c_{1}^{*} & 0 \\
0 & 0 & 1
\end{array}\right\| ; T_{2}^{*}=\left\|\begin{array}{ccc}
0 & -1 & 0.4 \\
1 & 0 & 0 \\
0 & 0 & 1
\end{array}\right\| ; T_{3}^{*}=\left\|\begin{array}{ccc}
-c_{1}^{*} & -s_{1}^{*} & 0.2 \\
s_{1}^{*} & -c_{1}^{*} & 0 \\
0 & 0 & 1
\end{array}\right\| ; \\
{ }^{0} \mathbf{r}_{D}=\left\|\begin{array}{l}
0.2 \\
0 \\
1
\end{array}\right\| ; \quad{ }^{3} \mathbf{r}_{D}=\left\|\begin{array}{l}
0.4 \\
0 \\
1
\end{array}\right\| .
\end{gathered}
$$

By writting the equation (1.5) for the point $D$, we get

$$
\left\|\begin{array}{c}
0.2 \\
0 \\
1
\end{array}\right\|=T_{1}^{*} T_{2}^{*} T_{3}^{*}\left\|\begin{array}{c}
0.4 \\
0 \\
1
\end{array}\right\|=\left\|\begin{array}{c}
-0.2 s_{1}^{*}+0.4 c_{1}^{*} \\
0.4 s_{1}^{*}+0.2 c_{1}^{*}-0.4 \\
1
\end{array}\right\| .
$$

Since we obtain two equations, that are

$$
-0.2 s_{1}^{*}+0.4 c_{1}^{*}=0.2 ; \quad 0.4 s_{1}^{*}+0.2 c_{1}^{*}=0.4 .
$$


By solving these equations, we get

$$
c_{1}^{*}=0.8 ; \quad s_{1}^{*}=0.6 .
$$

In order define the velocity and acceleration, let us to determine the following matrices.

$$
\begin{aligned}
& T_{1}^{\prime *}=\left\|\begin{array}{ccc}
-0.6 & -0.8 & 0 \\
0.8 & -0.6 & 0 \\
0 & 0 & 0
\end{array}\right\| ; T_{2}^{\prime *}=\left\|\begin{array}{ccc}
-1 & 0 & 0 \\
0 & -1 & 0 \\
0 & 0 & 0
\end{array}\right\| ; T_{3}^{\prime *}=\left\|\begin{array}{ccc}
-0.6 & 0.8 & 0 \\
-0.8 & -0.6 & 0 \\
0 & 0 & 0
\end{array}\right\| \text {; } \\
& \boldsymbol{T}_{1}^{\prime \prime *}=\left\|\begin{array}{ccc}
-0.8 & 0.6 & 0 \\
-0.6 & -0.8 & 0 \\
0 & 0 & 0
\end{array}\right\| ; T_{2}^{\prime \prime *}=\left\|\begin{array}{ccc}
0 & 1 & 0 \\
-1 & 0 & 0 \\
0 & 0 & 0
\end{array}\right\| ; \quad T_{3}^{\prime \prime *}=\left\|\begin{array}{ccc}
0.8 & 0.6 & 0 \\
-0.6 & 0.8 & 0 \\
0 & 0 & 0
\end{array}\right\| ; \\
& \boldsymbol{v}_{1}^{*}=T_{1}^{* *} T_{2}^{*} T_{3}^{*} \mathbf{r}_{\mathrm{D}}=\left\|\begin{array}{l}
0 \\
0.2 \\
0
\end{array}\right\| ; \quad \boldsymbol{v}_{2}^{*}=\mathbf{T}_{1}^{*} T_{2}^{*} T_{3}^{*} \mathbf{r}_{D}=\left\|\begin{array}{c}
0.24 \\
-0.12 \\
0
\end{array}\right\| \\
& \boldsymbol{v}_{3}^{*}=\boldsymbol{T}_{1}^{*} T_{2}^{*} \mathbf{T}_{\mathbf{3}}^{* \mathbf{3}} \mathbf{r}_{D}=\left\|\begin{array}{l}
0.4 \\
0 \\
0
\end{array}\right\|
\end{aligned}
$$

The point $D$ is fixed with respect to the global reference. Therefore their components of velocity and acceleration in the global reference are equal to zero. By writting the velocity's equation (the equation (1.7)) for the point $D$ at the given instant of time and developping it we obtain

$$
0=0.24 \dot{\varphi}_{2}^{*}+0.4 \dot{\varphi}_{3}^{*} ; \quad 0=0.2 \dot{\varphi}_{1}^{*}-0.12 \dot{\varphi}_{2}^{*}
$$

Solving these equations we get

$$
\dot{\varphi}_{2}^{*}=\frac{5}{3} \dot{\varphi}_{1}^{*}=\frac{5}{3} \omega_{1} ; \quad \dot{\varphi}_{3}^{*}=-\frac{3}{5} \dot{\varphi}_{2}^{*}=-\dot{\varphi}_{1}^{*}
$$

The absolute angular velocities of the links $\mathrm{BC}$ and $\mathrm{CD}$ will be

$$
\Omega_{2}^{*}=\dot{\varphi}_{1}^{*}+\dot{\varphi}_{2}^{*}=\frac{8}{3} \dot{\varphi}_{1}^{*}=\frac{8}{3} \omega_{1} ; \Omega_{3}^{*}=\dot{\varphi}_{1}^{*}+\dot{\varphi}_{2}^{*}+\dot{\varphi}_{3}^{*}=\frac{5}{3} \omega_{1} .
$$

In order to determine the acceleration let us write the matrix equation (1.12). For this aim, it is necessary to define the matrices $\boldsymbol{v}_{1}^{\prime}, \boldsymbol{v}_{2}^{\prime}, \boldsymbol{v}_{3}^{\prime}, \boldsymbol{v}_{1,2}, \boldsymbol{v}_{1,3}, \boldsymbol{v}_{2,3}$ at the given instant of time.

It is easy to obtain

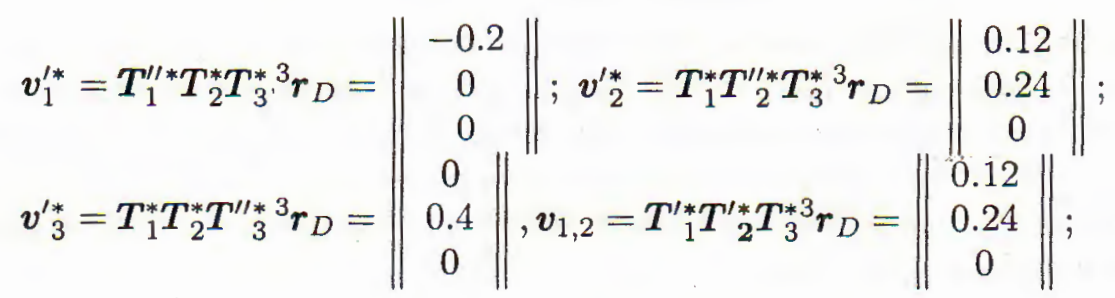




$$
\boldsymbol{v}_{1,3}=T_{1}^{* *} T_{2}^{*} T_{3}^{\prime * 3} r_{D}=\left\|\begin{array}{c}
0 \\
0.4 \\
0
\end{array}\right\| ; v_{2,3}=T_{1}^{*} T_{2}^{\prime *} T_{3}^{* 3} r_{D}=\left\|\begin{array}{c}
0 \\
0.4 \\
0
\end{array}\right\|
$$

Writting the equation (1.12) and developping it we get

$$
\begin{aligned}
& { }^{0} \ddot{x}_{D}=0 \rightarrow 0.24 \ddot{\varphi}_{2}^{*}+0.40 \ddot{\varphi}_{3}^{*}-0.20 \dot{\varphi}_{1}^{2 *}+0.12 \dot{\varphi}_{2}^{2 *}+0.24 \dot{\varphi}_{1}^{*} \dot{\varphi}_{2}^{*}, \\
& { }^{0} \ddot{y}_{D}=0 \rightarrow 0.20 \ddot{\varphi}_{1}^{*}-0.12 \ddot{\varphi}_{2}^{*}+0.24 \dot{\varphi}_{2}^{2 *}+0.40 \dot{\varphi}_{3}^{2 *}+0.48 \dot{\varphi}_{1}^{*} \dot{\varphi}_{2}^{*} .
\end{aligned}
$$

By substituting

$$
\dot{\varphi}_{1}^{*}=\omega_{1}, \ddot{\varphi}_{1}^{*}=\varepsilon_{1} ; \dot{\varphi}_{2}^{*}=\omega_{2}=\frac{5}{3} \omega_{1} ; \dot{\varphi}_{3}^{*}=-\omega_{1}, \varepsilon_{2}=\dot{\varphi}_{1}^{*}+\dot{\varphi}_{2}^{*} ; \varepsilon_{3}=\ddot{\varphi}_{1}^{*}+\ddot{\varphi}_{2}^{*}+\ddot{\varphi}_{3}^{*},
$$

into the above equations and solving them we will determine the angular accelerations of the connecting rod $B C$ and the bar $D C$, that are

$$
\varepsilon_{2}=\frac{8}{3} \varepsilon_{1}-\frac{20}{9} \omega_{1}^{2} ; \varepsilon_{3}=\frac{5}{3} \varepsilon_{1}-\frac{20}{9} \omega_{1}^{2} .
$$

\section{CONCLUSION}

The proposed method allows to define the configuration of mechanisms by means of the point of general view. It is important that by such a way it is possible to apply directly and usefully the softwere as MATLAB, MAPLE, MATCAD,... for solving complecated systems.

This work is completed by financial support of the Basic Program in Natural Science

\section{REFERENCES}

1. H. Abdellatif, M. Grotjahn, and B. Heimann, High Efficient Dynamics Calculation Aproach for Computed-Force Control of Robots with Parallel Structures, Proc. of the $44^{\text {th }}$ IEEE Conference on Decision and Control and the 2005 European Control Conference, CDC-ECC05, Sevilla, Spain, 2005, pp.2024-2029.

2. H. Cheng, Y. Kuen, and Z. Li, Dynamimics and Control of Redundantly Actuated Parallel Manipulators, IEEE/ASME Transactions on Mechatronics 8 (4) (2003) 483-491.

3. Do Dang Khoa, 'Tran Duc, Dinh Van Phong, Do Sanh, The Optimal Control for the System of Program Motion, The $3^{\text {rd }}$ Vietnam Conference on Mechatronics, VMC 2006, Hanoi, Vietnam, Publ. Hanoi National University, pp. 87-95.

4. E. J. Haug, Computer Aided Kinematics and Dynamics of Mechanical Systems, Volume I, Basic Method, Alyn\& Bacon, NewYork, 1989.

5. E. NikravesP, Computer Aided Analysics of Mechanical System, Printice Hall, NewYork, 1989.

6. B. Paul, Kinematics and Dynamics of Planar Machinery, Printice Hall, NewYork,1989.

7. A. Shabana, Dynamics of Multibody Systems, John Willey\&Sonc, NewYork/Chichester/Brisbane, 1989.

Received October 4, 2006

\section{PHƯONG PHÅP MA TRẠN TRUYỀN KHẢO SÁT CÁC CƠ CẨU PHÄNG}

Trong bài báo đề xuất một phương pháp khảo sát động học các cơ cấu phẳng, bài toán đang được quan tâm do đòi hỏi của phát triển kỹ thuật, đặc biệt trong các bài toán tổng hợp và điều khiển chuyển động. Trong phương pháp đề xuất đã xây dựng một lộ trình đơn giản để xác định các yếu tố hình - động học của cơ cấu (vị trí, vận tốc, gia tốc). Đặc biệt theo lộ trình được nêu cho phép sử dụng tiện lợi các phần mềm chuyên dụng như MATCAD, MAPLE, để giải quyết các bài toán động học các cơ cấu phẳng. 